\title{
SHORT MODULES AND ALMOST NOETHERIAN MODULES
}

\author{
G. BILHAN and P. F. SMITH
}

\begin{abstract}
It is proved that, for any ring $R$, a right $R$-module $M$ has the property that, for every submodule $N$, either $N$ or $M / N$ is Noetherian if and only if $M$ contains submodules $K \supseteq L$ such that $M / K$ and $L$ are Noetherian and $K / L$ is almost Noetherian.
\end{abstract}

\section{Short modules and almost Noetherian modules}

All rings are associative with identity and all modules are unital right modules. Let $R$ be any ring. In [11], Sarath defines an $R$-module $M$ to be tall if $M$ contains a submodule $N$ such that $N$ and $M / N$ are both non-Noetherian. We shall call an $R$-module short if it is not tall. Thus a module $M$ is short if and only if, for each submodule $N$ of $M$, either $N$ or $M / N$ is Noetherian. Clearly every Noetherian module is short. As we shall see below, it is easy to produce examples of short modules which are not Noetherian.

Following [2], we call an $R$-module $M$ almost Noetherian if every proper submodule of $M$ is finitely generated. Clearly a module $M$ is almost Noetherian if every proper submodule of $M$ is Noetherian. Clearly also, almost Noetherian modules are short. It is proved in [6, Theorem 2.2] that if $Z$ is the ring of rational integers then a Z-module $M$ is almost Noetherian if and only if $M$ is Noetherian or is isomorphic to the Prüfer $p$-group $\mathbf{Z}\left(p^{\infty}\right)$ for some prime $p$. In [2, Theorem 2.1], Armenderiz characterized all commutative rings $R$ such that the ring of fractions of $R$ is an almost Noetherian $R$-module. In particular, if $R$ is a discrete valuation ring then the field of fractions $K$ of $R$ is an almost Noetherian $R$-module, so that $K$ is a short $R$-module which is not Noetherian.

In [13], an $R$-module $M$ is called almost finitely generated (a.f.g.) if $M$ is not finitely generated as an $R$-module but every proper $R$-submodule of $M$ is finitely generated. (Note that in [5], a.f.g. modules are called "Jónsson $w_{0^{-}}$ generated modules".) Weakley [13] proved that if $R$ is a commutative ring and $M$ is an a.f.g. $R$-module then $P=\{r \in R: r M=0\}$ is a prime ideal of $R$.

Received April 8, 2004. 
Moreover, the $(R / P)$-module $M$ is divisible and is either torsion or torsionfree. If $M$ is a torsion-free $(R / P)$-module then $M$ is isomorphic to the field of fractions of the domain $R / P$. Otherwise, $M$ is an Artinian module such that $M \cong M / N$ for every proper submodule $N$ of $M$. For more information on a.f.g. modules see [5], [8] and [13].

Note the following elementary result.

Proposition 1.1. Let $R$ be any ring and let $M$ be an Artinian $R$-module. Then $M$ contains an almost Noetherian submodule.

Proof. Suppose that $M$ is not Noetherian. Then the non-empty collection of submodules $L$ of $M$ such that $L$ is not Noetherian has a minimal member $N$. Clearly $N$ is almost Noetherian.

We mention next two related results. The first is simply a restatement of [11, Theorem 2.7]. For the definition and properties of Krull dimension see [9, Chapter 6].

Proposition 1.2. The following statements are equivalent for a ring $R$.

(i) Every right R-module with Krull dimension is Noetherian.

(ii) Every short right $R$-module is Noetherian.

(iii) Every almost Noetherian right $R$-module is Noetherian.

A ring $R$ is called a right $V$-ring if every simple right $R$-module is injective. Kaplansky proved that a commutative ring is a (right) $V$-ring if and only if $R$ is von Neumann regular (see [10, Theorem 6]). Yousef [14, Theorem 1] proved that if $R$ is a right $V$-ring then every right $R$-module with Krull dimension is Noetherian. By Proposition 1.2 it follows that if $R$ is a right $V$-ring then every short right $R$-module is Noetherian. Bass [3, Theorem P] proved that if $R$ is a right perfect ring then every non-zero right $R$-module contains a maximal submodule and hence every almost Noetherian module is Noetherian. By Proposition 1.2 it follows that if $R$ is a right perfect ring then every short right $R$-module is Noetherian.

For commutative rings we have the following result characterising when every short module is Noetherian.

Proposition 1.3. The following statements are equivalent for a commutative ring $R$.

(i) Every short R-module is Noetherian.

(ii) Every Artinian module is Noetherian.

(iii) No homomorphic image of $R$ is isomorphic to a dense subring of a complete local Noetherian domain of dimension 1. 
Proof. By Proposition 1.2, [1, Theorem 3.10(ii)] and [4, Proposition 4.4].

Let $R$ be any commutative Noetherian domain which is not a field. Let $P$ be maximal in the collection of non-maximal prime ideals of $R$. Then $R / P$ is a one-dimensional Noetherian domain. The $\operatorname{ring} R / P$ is isomorphic to a dense subring of a complete local Noetherian domain of dimension 1. By Proposition 1.3, there exist short $R$-modules which are not Noetherian.

In general, not every short module is almost Noetherian. This is a consequence of the following result.

Lemma 1.4. Let $R$ be any ring. Then any extension of a Noetherian $R$ module by a short $R$-module is short.

Proof. Let $K$ be a Noetherian submodule of an $R$-module $M$ such that $M / K$ is short. Let $N$ be any submodule of $M$. Then $N \cap K$ is Noetherian. If $N /(N \cap K)$ is Noetherian then so too is $N$. Suppose that $N /(N \cap K)$ is not Noetherian. Then $(N+K) / K$ is a non-Noetherian submodule of the short module $M / K$. It follows that $M /(N+K)$ is Noetherian. But $(N+K) / N \cong$ $K /(N \cap K)$ which is Noetherian. Thus $M / N$ is Noetherian.

COROLlary 1.5. Let $R$ be any ring and let an $R$-module $M=M_{1} \oplus M_{2}$ be a direct sum of a short submodule $M_{1}$ and a Noetherian submodule $M_{2}$. Then $M$ is short.

Proof. By Lemma 1.4.

We have already noted that a Z-module $M$ is almost Noetherian if and only if $M$ is Noetherian or $M$ is isomorphic to the Prüfer $p$-group $Z\left(p^{\infty}\right)$, for some prime $p$. By Lemma 1.4, for each prime $p$, the Z-module $M_{p}=$ $\left\{m / p^{n}: m, n \in \mathbf{Z}, n \geq 0\right\}$ is a short Z-module which is not almost Noetherian. Alternatively by Corollary 1.5 , the $\mathbf{Z}$-module $\mathbf{Z} \oplus \mathbf{Z}\left(p^{\infty}\right)$ is short but not almost Noetherian, for every prime $p$.

Lemma 1.6. Let $R$ be any ring. Then any extension of a short $R$-module by a Noetherian $R$-module is short.

Proof. Let $K$ be a submodule of an $R$-module $M$ such that $K$ is short and $M / K$ is Noetherian. Let $N$ be any submodule of $M$. Then $(N+K) / K$ is Noetherian and hence so too is $N /(N \cap K)$. If $N \cap K$ is Noetherian then so too is $N$. Suppose that $N \cap K$ is not Noetherian. By hypothesis, $K /(N \cap K)$ is Noetherian and hence $M /(N \cap K)$ is Noetherian. This implies that $M / N$ is Noetherian.

COROLlary 1.7. Let $R$ be any ring and let $M$ be an $R$-module such that $M$ contains submodules $N \supseteq L$ with $N / L$ short and both $M / N$ and $L$ Noetherian. Then $M$ is short. 
Proof. By Lemmas 1.4 and 1.6.

It is clear that non-Noetherian almost Noetherian modules do not contain maximal submodules. We show next that all other non-zero short modules do contain maximal submodules.

Proposition 1.8. Let $R$ be any ring and let $M$ be a non-zero short $R$ module. Then $M$ is almost Noetherian or M contains a maximal submodule.

Proof. Suppose that $M$ is not almost Noetherian. Then there exists a proper submodule $L$ of $M$ such that $L$ is not Noetherian. Because $M$ is short, the module $M / L$ is Noetherian and hence $M / L$ (and also $M$ ) contains a maximal submodule.

Recall that a module $M$ has finite uniform dimension if $M$ does not contain a direct sum of an infinite number of non-zero submodules.

Lemma 1.9. Let $R$ be any ring. Then any short $R$-module has finite uniform dimension.

Proof. Suppose that a module $M$ contains an infinite direct sum $N_{1} \oplus N_{2} \oplus$ $N_{3} \oplus \ldots$ of non-zero submodules $N_{i}(i \geq 1)$. Let $N=N_{1} \oplus N_{3} \oplus N_{5} \oplus \ldots$ Clearly the submodule $N$ is not Noetherian Moreover, $N_{2} \oplus N_{4} \oplus N_{6} \oplus \ldots$ embeds in $M / N$ so that the module $M / N$ is not Noetherian. Thus $M$ is not short.

Lemma 1.10. Let $R$ be any ring and let $M$ be a short $R$-module. Then every submodule and every homomorphic image of $M$ is short.

Proof. Clear.

It is not the case, in general, that the direct sum of two short modules is short. We observed above that, for any prime $p$, the $\mathbf{Z}$-module $\mathbf{Z}\left(p^{\infty}\right)$ is almost Noetherian and hence short but the Z-module $\mathbf{Z}\left(p^{\infty}\right) \oplus \mathbf{Z}\left(p^{\infty}\right)$ is clearly not short. We complete this section by characterising short modules in terms of almost Noetherian modules.

THeORem 1.11. Let $R$ be any ring. An $R$-module $M$ is short if and only if $M$ contains submodules $N \supseteq L$ such that $N / L$ is almost Noetherian and $M / N$ and $L$ are both Noetherian.

Proof. The sufficiency follows by Corollary 1.7. Conversely, suppose that $M$ is a non-Noetherian short module. There exists a submodule $H$ of $M$ such that $H$ is not finitely generated. By Lemmas 1.9 and 1.10 every factor module of $H$ has finite uniform dimension. Shock [12, Theorem 3.7] proved that a non-finitely generated module $X$, such that $Y / Z$ has a maximal submodule for 
all submodules $Y \supsetneqq Z$ of $X$, contains a submodule $U$ such that the module $X / U$ has non-finitely generated socle. Hence there exist submodules $N \supsetneqq L$ of $H$ such that the non-zero module $N / L$ does not contain a maximal submodule. But Lemma 1.10 gives that $N / L$ is short. By Proposition $1.8 N / L$ is almost Noetherian. Because $N / L$ is not Noetherian we have $N$ is not Noetherian and hence $M / N$ is Noetherian. Finally, because $N / L$ is not Noetherian we have $M / L$ is not Noetherian and hence $L$ is Noetherian. This completes the proof.

\section{Properties of short modules}

In this section we shall obtain some properties of short modules over an arbitrary ring $R$. The next result improves Lemma 1.9.

Proposition 2.1. Short modules have Krull dimension.

Proof. By the proof of [11, Theorem 2.7(i) $\Rightarrow$ (ii)].

For any ring $R, \operatorname{Soc}\left(R_{R}\right)$ will denote the right socle of $R$. Proposition 2.1 has the following consequence for rings.

Corollary 2.2. Let $S$ be a semiprime ring. Then the right $S$-module $S$ is short if and only if $S$ is right Noetherian.

Proof. The sufficiency is clear. Conversely, suppose that $S$ is a short $S$ module. If $S / E$ is a Noetherian module for every essential right ideal $E$ of $S$, Goodearl [7, Proposition 3.6] proved that the $\operatorname{ring} S / \operatorname{Soc}\left(S_{s}\right)$ is right Noetherian. But $\operatorname{Soc}\left(S_{s}\right)$ is Noetherian by Lemma 1.9. Thus $S$ is right Noetherian. Now suppose that there exists an essential right ideal $E^{\prime}$ of $S$ such that $S / E^{\prime}$ is not Noetherian. This implies that $E^{\prime}$ is a Noetherian $S$-module. By [9, Proposition 6.3.5] $S$ is a right Goldie ring and by [9, Proposition 2.3.5] there exists an element $c$ of $E^{\prime}$ such that $S \cong c S$. It follows that $S$ is a right Noetherian ring.

It is easy to give an example of a non-semiprime ring $S$ such that the right $S$-module $S$ is short but $S$ is not right Noetherian.

ExAmple 2.3. Let $p$ be any prime and let $S$ be the trivial extension of the $\mathrm{Z}$-module $\mathrm{Z}\left(p^{\infty}\right)$ by $\mathrm{Z}$. Then $S$ is a commutative ring such that the $S$-module $S$ is short but not Noetherian.

Proof. Note that $S$ consists of all ordered pairs $(a, m)$, where $a \in \mathbf{Z}, m \in$ $\mathbf{Z}\left(p^{\infty}\right)$, and addition and multiplication are defined by

$$
(a, m)+\left(a^{\prime}, m^{\prime}\right)=\left(a+a^{\prime}, m+m^{\prime}\right), \text { and }(a, m)\left(a^{\prime}, m^{\prime}\right)=\left(a a^{\prime}, a m^{\prime}+a^{\prime} m\right)
$$

for all $a, a^{\prime} \in \mathbf{Z}, m, m^{\prime} \in \mathbf{Z}\left(p^{\infty}\right)$. It is easy to check that $S$ is a commutative ring and that $I=\left\{(0, m): m \in \mathbf{Z}\left(p^{\infty}\right)\right\}$ is an ideal of $S$ such that $S / I \cong \mathbf{Z}$. Let 
$J$ be an ideal of $S$. Because $\mathrm{Z}\left(p^{\infty}\right)$ is a divisible Abelian group, one can easily check that $J \subseteq I$ or $I \subseteq J$. If $J \varsubsetneqq I$ then $J$ is finite and hence Noetherian. If $I \subseteq J$ then $S / J$ is Noetherian. Thus $S$ is a short $S$-module. That $S$ is not Noetherian is clear because $I$ is not a finitely generated ideal.

Proposition 2.4. Short modules are countably generated.

Proof. Let $M$ be a short module over a ring $R$. Suppose that $M$ is not finitely generated. Let $0 \neq m_{1} \in M$. Because $M \neq m_{1} R$, there exists $m_{2} \in$ $M \backslash m_{1} R$. Also because $M \neq m_{1} R+m_{2} R$, there exists $m_{3} \in M \backslash\left(m_{1} R+m_{2} R\right)$. This process produces a proper ascending chain $m_{1} R \varsubsetneqq m_{1} R+m_{2} R \varsubsetneqq \cdots$ of submodules of $M$. Let $N=\sum_{n=1}^{\infty} m_{n} R$. Clearly the submodule $N$ is not Noetherian. Thus $M / N$ is Noetherian and in particular $M / N=\left(x_{1}+N\right) R+$ $\ldots+\left(x_{k}+N\right) R$ for some positive integer $k$ and elements $x_{i} \in M(1 \leq i \leq k)$. Finally $M=\sum_{n=1}^{\infty} m_{n} R+\sum_{i=1}^{k} x_{i} R$.

Proposition 2.5. Let $K, L$ be submodules of a short module $M$ such that $M=K+L$. Then there exists a finitely generated submodule $K_{1}$ of $K$ such that $M=K_{1}+L$ or there exists a finitely generated submodule $L_{1}$ of $L$ such that $M=K+L_{1}$.

Proof. If $K$ is finitely generated then set $K_{1}=K$. Suppose that $K$ is not finitely generated. Because $M$ is short, the module $M / K$ is finitely generated. But $M / K \cong L /(L \cap K)$, so that $L /(L \cap K)$ is finitely generated. It follows that there exists a finitely generated submodule $L_{1}$ of $L$ such that $L=L_{1}+(L \cap K)$. In this case, $M=L+K=L_{1}+(L \cap K)+K=L_{1}+K$.

A module $M$ is called locally Noetherian provided every finitely generated submodule of $M$ is Noetherian. For example, every right module over a right Noetherian ring is locally Noetherian and so too is any semisimple module over an arbitrary ring.

Proposition 2.6. A short module is finitely generated or locally Noetherian.

Proof. Suppose that $M$ is not finitely generated. Let $N$ be any finitely generated submodule of $M$. Clearly $M / N$ is not finitely generated so that, by hypothesis, $N$ is Noetherian.

COROLlaRY 2.7. Let $M$ be a short module which is not finitely generated and let $K, L$ be submodules of $M$. Then $K+L$ is finitely generated if and only if both $K$ and $L$ are finitely generated.

Proof. The sufficiency is clear and the necessity follows by Proposition 2.6.

Proposition 2.8. Let $M$ be a short module and let $K, L$ be submodules of $M$ such that $K \cap L$ is finitely generated. Then either $K$ or $L$ is finitely generated. 
Proof. Suppose that $K$ and $L$ are not finitely generated. It follows that $L$ is not Noetherian and hence $M / L$ is Noetherian. Because $K /(K \cap L) \cong$ $(K+L) / L$, the module $K /(K \cap L)$ is finitely generated. Finally $K$ not finitely generated implies that $K \cap L$ is not finitely generated. The result follows.

Note that if $M$ is a short module which is not finitely generated and $K$ is a finitely generated submodule of $M$ then Proposition 2.6 gives that $K \cap L$ is finitely generated for any submodule $L$ of $M$, i.e. the converse of Proposition 2.8 holds.

\section{REFERENCES}

1. Albu, T., and Smith, P. F., Global Krull dimension, in Interactions between ring theory and representations of algebras, eds F. van Oystaeyen and M. Saorin, Dekker, New York (2000), $1-21$.

2. Armendariz, E. P., Rings with an almost Noetherian ring of fractions, Math. Scand. 41 (1977), $15-18$.

3. Bass, H., Finitistic dimension and a homological generalization of semiprimary rings, Trans. Amer. Math. Soc. 95 (1960), 466-488.

4. Facchini, A., Loewy and Artinian modules over commutative rings, Ann. Mat. Pura Appl. (4) 128 (1981), 359-374.

5. Gilmer, R., and Heinzer, W., Cardinality of generating sets for modules over a commutative ring, Math. Scand. 52 (1983), 41-57.

6. Gilmer, R., and O'Malley, M., Non-Noetherian rings for which each proper subring is Noetherian, Math. Scand. 31 (1972), 118-122.

7. Goodearl, K. R., Singular torsion and the splitting properties, Mem. Amer. Math. Soc. 124 (1972).

8. Heinzer, W., and Lantz, D., Artinian modules and modules of which all proper submodules are finitely generated, J. Algebra 95 (1985), 201-216.

9. McConnell, J. C., and Robson, J. C., Noncommutative Noetherian rings, Wiley-Interscience, Chichester, 1987.

10. Rosenberg, A., and Zelinsky, D., On the finiteness of the injective hull, Math. Z. 70 (1959), 372-380.

11. Sarath, B., Krull dimension and Noetherianness, Illinois J. Math. 20 (1976), 329-335.

12. Shock, R. C., Dual generalizations of the Artinian and Noetherian conditions, Pacific J. Math. 54 (1974), 227-235.

13. Weakley, W. D., Modules whose proper submodules are finitely generated, J. Algebra 84 (1983), 189-219.

14. Yousif, M. F., V-modules with Krull dimension, Bull. Austral. Math. Soc. 37 (1988), 237-240.

DOKUZ EYLÜL UNIVERSITY

DEPARTMENT OF MATHEMATICS

KAYNAKLAR Y. BUCA-IZMIR

TÜRKIYE

E-mail: gokhan.bilhan@deu.edu.tr
DEPARTMENT OF MATHEMATICS UNIVERSITY OF GLASGOW

GLASGOW, G12 8QW

SCOTLAND, UK

E-mail: pfs@maths.gla.ac.uk 